\title{
SOME PROBLEMS OF DIFFERENCE SCHEME THEORY*
}

\author{
A. A. SAMARSKII \\ Moscow \\ (Received 28 January 1966)
}

1. DIFFERENCE schemes for solving various problems of mathematical physics have appeared in recent years. Economic methods for solving multidimensional problems have been particularly developed (alternating direction schemes, decomposition schemes, locally one-dimensional schemes etc.). A large number of different algorithms is now available for solving a given problem. Special interest is thus aroused by the theoretical and experimental comparison of difference schemes, the development of general principles for constructing families of difference schemes possessing given properties, and finding the schemes in the family that satisfy auxiliary optimality requirements (as regards accuracy, economy etc.).

There are various schemes which give the same results (are algebraically identical) provided the right-hand sides and boundary conditions are matched in a certain way. In this case our choice of one scheme rather than another must be based on practical convenience. This situation is typical for economic methods of solving multidimensional problems. Several papers $[1,2,3,4]$ have been concerned with comparing economic methods.

A comparison in $[1,2]$ with the operator decomposition method (for the equation of heat conduction in a rectangular region), to which the methods of [5 - 7] are reducible after eliminating the intermediate values, snowed that the order of approximation of these methods depends, in the case of non-stationary boundary conditions (and for [7], in the case of stationary conditions also) on the method of specifying the boundary conditions at the intermediate step. It is shown that, by varying the right-hand side at the boundary base-points of the mesh, we can

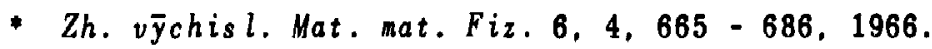


arrange for method [5] to be the same as the operator decomposition method $O\left(\tau^{2}+h^{2}\right)$. In [7], boundary conditions are written for the method of [5] for the intermediate value $y^{n+1 / 2}$, leading to the same result. If the right-hand sides or boundary conditions are suitably matched, the methods of $[2,5,7]$ and certain others can be treated, as shown in $[3,4]$, as computational algorithms realizing the same scheme with split operator. All these schemes are algebraically identical.

Additive (locally one-dimensional) schemes forming a sequence of intermediate (in the elementary case one-dimensional) schemes were considered in [8] for the parabolic equation in Banach space with the operator

$$
A=\sum_{\alpha=1}^{\rho} A_{\alpha} \text { or } \quad A=\sum_{\alpha=1}^{\infty} A_{\alpha 3},
$$

where $A_{\alpha}, A_{\alpha \beta}$ are operators of simpler structure than $A$. For the additive scheme, the error of approximation is the sum of the approximation errors [9] for all the intermediate schemes (additivity of the approximation). From this point of view, the alternating-direction-implicit (ADI) scheme of [5] is a locally one-dimensional scheme of a special kind.

2. A basic problem in difference scheme theory is to find sufficient conditions for stability.

If we investigate difference schemes as operator equations in austract space $H$ with a scalar product, we can use the method of energy inequalities [10] to obtain sufficient conditions for two-layer and multi-layer schemes in a form that can easily be checked. We only make use here of general information on the operators such as positiveness (semiboundedness from below) and selfadjointness. For instance, the the two-layer scheme

$$
\begin{aligned}
& B y_{\vec{t}}+A y=0, \quad 0<t=j \tau, \quad y(0)=y_{0}, \quad y_{t}=(y-\tilde{y}) / \tau, \\
& y=y^{j+1}, \quad \check{y}=y^{j}
\end{aligned}
$$

where $A$ and $B$ are linear operators in $H$, and $\tau>0$ is a constant, the sufficient conditions for stability, with which $\left\|y^{j}\right\|_{a} \leqslant\left\|y_{0}\right\|_{a}\left(\|y\|_{a}=\right.$ $\sqrt{(A y, y)}, A$ is independent of $t)$, are

$$
(B y, y) \geqslant 0.5 \tau(A y, y), \quad(A y, y) \geqslant 0, \quad(B y, y) \geqslant 0
$$

if $A$ is selfadjoint. Hence, in particular, we obtain for the scheme

$$
y_{\vec{t}}+A(\sigma y+(1-\sigma) y)=0, \quad 0<t=j \tau, \ldots, \quad y(0)=y_{0},
$$


the sufficient condition for stability $\sigma \geqslant 0.5-1 / \tau\|A\|$, since $\mathrm{B}=$ $E+\sigma \tau A$.

More exact stability conditions involving the initial data are required to prove the convergence of iterational schemes.

3. In the present paper, we illustrate the above topics of the general theory (algebraic identity and stability) from the example of the iterational schemes for solving the stationary equation $\left(A_{1}+A_{2}\right) v=f$, where $A_{1}, A_{2}$ are linear positive-definite operators in real Hilbert space $H$.

We show in Section 1 that the ADI scheme is algebraically identical with three extremely simple locally one-dimensional schemes.

The rate of convergence of the ADI scheme was investigated in [11] for the case when $A_{1}$ and $A_{2}$ are arbitrary positive definite operators.

In Section 2, which is a development of [11], we obtain new estimates of the rate of convergence of the $A D I$ scheme. In addition to assuming that $A_{1}$ and $A_{2}$ are positive definite,

$$
(A y, y) \geqslant \delta\|y\|^{2}, \quad \delta=\text { const }>0,
$$

we use the condition

$$
\|A y\|^{2} \leqslant \Delta(A y, y), \quad \Delta=\text { const }>0
$$

which involves no loss of generality, since, for any positive definite operator $A$,

$$
\Delta \leqslant \frac{1}{8}\|A\|^{2} \quad\left(\Delta=\|A\| \text { if } A^{*}=A\right)
$$

In Section 3, we show by examples that $\Delta$ is easily evaluated for certain non-selfadjoint operators.

When assessing the rate of convergence of the LTS, the main role is played by a bound on the norm of the transition operator (Theorem 5):

$$
\begin{aligned}
& \left\|(E+\sigma \tau A)^{-1}(E-(1-\sigma) \tau A)\right\| \leqslant \sqrt{\rho}, \\
& \rho=\frac{1-2(1-\sigma) \tau \delta+(1-\sigma)^{2} \tau^{2} \delta \Delta}{1+2 \sigma \tau \delta+\sigma^{2} \tau^{2} \delta \Delta},
\end{aligned}
$$

which implies, in particular, the stability condition $\sigma \geqslant 0.5-1 / \tau \Delta$ for the above-mentioned schemes with weights, without the assumption 
that $A$ is selfadjoint.

We show that, when using the ADI scheme, the initial error can be reduced in the ratio $1 / \varepsilon$ ( $\varepsilon>0$ is the given accuracy) simply by performing

$$
v(\varepsilon) \geqslant \ln \frac{1}{8}\left[\ln \frac{1+\sqrt{\eta}}{1-\sqrt{\eta}}\right]^{-1}
$$

iterations, where $\eta=\delta / \Delta\left(\delta=\min \left(\delta_{1}, \delta_{2}\right), \Delta=\max \left(\Delta_{1}, \Delta_{2}\right)\right.$ with $T=1 / \sqrt{\delta \Delta}$.

Notice that other authors (e.g. [14, 15, 17, 18]) studied the convergence of the ADI scineme on the assumption that $A_{1}$ and $A_{2}$ are finitedimensional, positive definite, selfadjoint and conmutative.

Some applications of the general theory of Section 2 are also considered in Section 3 .

We show that the ADI scheme is convergent for the Dirichlet difference problem in an arbitrary region, whether the elliptic operator with variable coefficients on arbitrary meshes is selfadjoint or not. It follows from the bound for $v(\varepsilon)$ that $v(\varepsilon) \approx 1 / h$ ln $1 / \varepsilon$ as $h \rightarrow 0$, where $h$ is the mesh interval.

We also consider the application of the general theory to the transport equation. An iterational scheme was proposed in $[12,13]$ which may be seen from Section 1 to be algebraically identical with the ADI scheme. Hence its convergence for any $\tau$, and the estimate of its rate of convergence, follow from [11] (we only need to use the fact that $A_{1}$ and $A_{2}$ are positive definite, and evaluate $\left.\left\|A_{1}\right\|,\left\|A_{2}\right\|\right)$. The convergence rate estimate of [11] can be improved by using Theorem 5 (of Section 1) while recalling that $A_{2}$ is selfadjoint.

Comparison with the method of simple iteration shows that extra study is needed before we can say that the ADI scheme is worth using as an economic algorithm for the radiation transport equation.

In paragraph 4 of Section 3 we consider the ADI scheme for solving the Dirichlet difference problem in an arbitary p-dimensional region, obtained by splitting the Laplace difference operator into a sum of nonselfadjoint operators. V.K. Saul'ev used this scheme (with $p=2$ and a rectangular region) for the equation of heat conduction (alternating method). We show that, in this case also, $v(\varepsilon)=O(1 / h \ln 1 / \varepsilon)$. 
We have confined ourselves to a few examples in order to illustrate how the convergence rate estimates of Section 2 should be applied (though the results of Section 3 are of independent interest).

\section{Comparison of various iterational schemes}

Given the equation

$$
\left(A_{1}+A_{2}\right) v=f
$$

where $A_{1}, A_{2}$ are linear operators in real Hilbert space $H$. We can solve it by using the iterational ADI scheme

$$
\begin{gathered}
\left(E+\tau A_{1}\right) \bar{y}=\left(E-\tau A_{2}\right) y^{k}+\tau f, \\
\left(E+\tau A_{2}\right) y^{t+1}=\left(E-\tau A_{1}\right) \bar{y}+\tau f, \quad k=0,1,2, \ldots, \\
y^{0}=y_{0} \in H,
\end{gathered}
$$

where $E$ is the unit operator, $k$ the number of iterations, $\bar{y}=y^{k+1 / 2}$ the intermediate value (subiteration), and $T$ the relaxation parameter ( $\tau=\tau_{k}$ is in general dependent on $k$ ). In addition to (2), we consider the scheme

$$
\begin{gathered}
y_{(1)}=\left(E-\tau A_{2}\right) y^{k}+\tau f_{1}, \quad\left(E+\tau A_{1}\right) y_{(2)}=y_{(1)}+\tau\left(f-f_{1}\right), \\
y_{(3)}=\left(E-\tau A_{1}\right) y_{(2)}+\tau f_{2}, \quad\left(E+\tau A_{2}\right) y^{k+1}=y_{(3)}+\tau\left(f-f_{2}\right), \\
k=0,1, \ldots, \quad y^{0}=y_{0},
\end{gathered}
$$

where $f_{1}$ and $f_{2}$ are arbitrary elements of $H$.

Whatever $f_{1}, f_{2}$, scheme (3) is algebraically identical with scheme (2), i.e. the $y^{k+1}$ found from (2) and (3) are the same. For, if we add the first to the second of equations (3), and the third to the fourth, we can eliminate $y_{(1)}, y_{(3)}$ and obtain equation (2) with $\bar{y}=y_{(2)}$.

We show that the schemes

$$
\bar{y}_{(1)}=\left(E-\tau A_{2}\right) \bar{y}^{k}+\tau f_{1}, \quad\left(E+\tau A_{1}\right) \bar{y}_{(2)}=\left(E-\tau A_{1}\right) \bar{y}_{(1)}+2 \tau\left(f-f_{1}\right),
$$$$
\left(E+\tau A_{2}\right) \bar{y}^{h+1}=\bar{y}_{(2)}+\tau f_{1}, \quad k=0,1,2, \ldots, \quad \bar{y}^{0}=y_{0},
$$ 
and

$$
\begin{gathered}
\tilde{y}_{(1)}=\left(E-\tau A_{2}\right) \tilde{y}^{k}+\tau f_{1}, \quad \tilde{y}_{(2)}=\left(E-\tau A_{1}\right) \tilde{y}_{(1)}+\tau \tilde{f}_{2}, \\
\left(E+\tau A_{1}\right) \tilde{y}_{(3)}=\tilde{y}_{(2)}+\tau\left\lfloor 2\left(f-f_{1}\right)-\tilde{f}_{2}\right], \quad\left(E+\tau A_{2}\right) \tilde{y}^{k+1}=\tilde{y}_{(3)}+\tau f_{1}, \\
\tilde{y}^{0}=y_{0} .
\end{gathered}
$$

are algebraically identical with scheme (2). For this, we only need to show that (4) and (5) are equivalent to scheme (3) with $f_{2}=f_{1}$, provided $\bar{y}^{k}=y^{k}, \tilde{y}^{k}=y^{k}$. Comparing (4) and (3), we see that the first and last equations of (3) and (4) are the same, $\bar{y}_{(1)}=y_{(1)}$. We show that $\bar{y}_{(2)}=y_{(3)}$. Introducing $\bar{y}=0.5\left(\bar{y}_{(1)}+\bar{y}_{(2)}\right)$, we can replace the second equation of (4) by the two equations

$$
\bar{y}_{(2)}=\left(E-\tau A_{1}\right) \bar{y}+\tau\left(f-f_{1}\right), \quad\left(E+\tau A_{1}\right) \bar{y}=\bar{y}_{(1)}+\tau\left(f-f_{1}\right) .
$$

The resulting $\bar{y}_{(2)}$ is the same as obtained from the second of equations (4). For, subtracting the second of equations (6) from the first, we get $\bar{y}=0.5\left(\bar{y}_{(1)}+\bar{y}_{(2)}\right)$. After substituting this expression in the first of equations (6), we get the second of equations (4).

We have thus proved the algebraic identity of (4) with (3), and hence with the ADI scheme (2).*

Adding the second and third of equations (5), we get

$$
\left(E+\tau A_{1}\right) \tilde{y}_{(3)}=\left(E-\tau A_{1}\right) \tilde{y}_{(1)}+2 \tau\left(f-f_{1}\right)
$$

Since $\tilde{y}_{(1)}=\bar{y}_{(1)}$ we have $\tilde{y}_{(3)} \doteq \bar{y}_{(2)}$ and schemes (5), (4) are identical.

Schemes (3), (4) and (5) are thus algebralcally identical with scheme (2), whatever the linear operators $A_{1}, A_{2}$ (this linearity was used in passing from (6) to the second of equations (4)). We are assuming here that $\left(E+\tau A_{\alpha}\right)^{-1}, \alpha=1,2$ exist. This is the only restriction on $A_{\alpha}$.

On the other hand, the solution of problem (2) satisfies

$$
\left(E+\tau A_{1}\right)\left(E+\tau A_{2}\right) y^{k+1}=\left(E-\tau A_{1}\right)\left(E-\tau A_{2}\right) y^{k}+2 \tau f
$$

- The equivalence of schemes (3) and (4) can be proved by eliminating the intermediate points $y_{(1)}, y_{(2)}, y_{(3)}$ in (3) and $\bar{y}_{(1)}, \bar{y}_{(2)}$ in (4). Our present method of proof, proposed by I.V. Fryazinov, is simpler. 
To prove this, we obtain from (2)

$$
2 \bar{y}=\left(E+\tau A_{2}\right) y^{k+1}+\left(E-\tau A_{2}\right) y^{k}
$$

and substitute this in the first or second of equations (2). If (7) has a unique solution, then (7), (2) are algebraically identical. For substituting $\left(E+\tau A_{2}\right) y^{k+1}=2 \bar{y}-\left(E-\tau A_{2}\right) y^{k}$ in (7), we obtain the first of equations (2); the second of equations (2) follows from this and from (8).

It was shown in $[1,4]$ that the decomposing operator scheme (7) is equivalent to several other schemes, in particular the ADI scheme. If $A_{1}, A_{2}$ are commutative, (7) is equivalent to the scheme $[3],[1,2]$

$$
\begin{gathered}
\left(E+\tau A_{1}\right) \bar{y}=\left(E-\tau A_{1}\right) y^{k}, \\
\left(E+\tau A_{2}\right) y^{k+1}=\left(E-\tau A_{2}\right) \bar{y}+2 \tau \varphi, \quad k=0,1, \ldots, \quad y^{0}=y_{0}
\end{gathered}
$$

where $\phi$ is the solution of the equation

$$
\left(E+\tau A_{1}\right) \varphi=f
$$

$[1,4]$

Thus there are many schemes equivalent to the ADI scheme (2).

When writing schemes in operator form, the question of non-homogeneous boundary conditions for the intermediate values $\bar{y}, y_{1}$ etc. does not arise, since the difference operators $A_{1}, A_{2}$ are now defined in the space of functions satisfying homogeneous boundary conditions, while the nonhomogeneous conditions are taken into account by the right-hand side.

The schemes considered above can also be used for solving non-stationary equations, e.g. the equation of heat conduction (with interval $2 \mathrm{~T}$ ).

Depending on the method of specifying the boundary conditions for the intermediate values $\left(\bar{y}, y_{(1)}, y_{(2)}\right.$ etc.), we obtain different schemes, which are only equivalent when the boundary conditions are matched $[1-4]$.

\section{Convergence of the iterational scheme}

1. Let $v$ be a solution of the initial problem (1), $y^{k+1}$ a solution of 
problem (2). For the difference $z^{k+1}=y^{k+1}-v$ the Cauchy problem

$$
\begin{gathered}
\left(E+\tau A_{1}\right) \bar{z}=\left(E-\tau A_{2}\right) z^{k}, \quad \bar{z}=\vec{y}-v, \quad z^{0}=y_{0}-v \\
\left(E+\tau A_{2}\right) z^{k+1}=\left(E-\tau A_{1}\right) \bar{z}, \quad k=0,1 \ldots \\
z^{0}=z_{0}=y_{0}-v .
\end{gathered}
$$

is obtained. To assess the rate of convergence of method (12), we need to know how fast the initial discrepancy $z_{0}=y_{0}-v$ is damped as the number of iterations increases. For this, we have to investigate the stability of problem (12) with respect to its initial data.

Let (,) be the scalar product, while $\|y\|=\sqrt{(y, y)}$ is the norm in real Hilbert space $H$.

We shall assume everywhere that the operators $A_{\alpha}$ are positive definite

$$
\left(A_{\alpha} y, y\right) \geqslant \delta_{\alpha}\|y\|^{2}, \quad \alpha=1,2, \quad \delta_{\alpha}=\text { const }>0, \quad y \in \mathrm{H} .
$$

The convergence of the ADI scheme was investigated in $[14-15]$ in complex finite - dimensional space $\tilde{H}_{N}$. By a theorem of functional analysis [16, Chap. V], if a linear operator specified in complex Hilbert space is positive, it must be selfadjoint.

The ADI scheme was applied in [11] as an economic method of solving systems of differential and algebraic equations. It was assumed that the matrix $A$ of the systen can be written as $A=A_{1}+A_{2}$, where $A_{1}, A_{2}$ are triangular matrices satisfying condition (13) only. The method of energy inequalities was used to prove the convergence, using only the fact that $A_{1}$ and $A_{2}$ are operators in a space with a scalar product satisfying (13).

Theorem 3 of [11] shows that the iterational scheme (2) is convergent at the rate of a geometric progression whatever $\tau>0$, the ratio $\rho$ of the progression being dependent on $\delta_{1}, \delta_{2}$ and $\left\|A_{1}\right\|,\left\|A_{2}\right\|$. It was shown how $T$ can be chosen so as to minimize $\rho$. The proof of Theorem 3 was performed for the case $D=E / T$, when equation (23) of [11] becomes (12).

To assist our future treatment, we state a corollary to Theorem 3 of [11] as a separate theorem, with a change of notation.

\section{Theorem 1}

If $A_{\alpha}$ are positive definite operators, then scheme (2) is convergent for any $T>0$, so that the bound

$$
\left\|z^{k+1}\right\|_{(2)} \leqslant \rho\left\|z^{k}\right\|_{(2)}, \quad k=0,1,2, \ldots \text {, }
$$


holds for the solution of problem (12), where

$$
\begin{gathered}
\|z\|_{(\alpha)}{ }^{2}=\|z\|^{2}+\tau^{2}\left\|A_{\alpha} z\right\|^{2}, \quad \rho^{2}=\rho_{1}{ }^{2} \rho_{2}{ }^{2}, \quad \rho_{\alpha}{ }^{2}=\frac{1-x_{\alpha}}{1+x_{\alpha}}, \\
x_{\alpha}=\frac{2 \tau \delta_{\alpha}}{1+\tau^{2}\left\|A_{\alpha}\right\|^{2}}, \quad \alpha=1,2 .
\end{gathered}
$$

The proof given in [11] is as follows. We evaluate the square of the norm of both sides of (12) and recall that

$$
\left\|\left(E \pm \tau A_{\alpha}\right) z\right\|^{2}=\|z\|_{(\alpha)}^{2} \pm 2 \tau\left(A_{\alpha} z, z\right), \quad 2 \tau\left(A_{\alpha} z, z\right) \geqslant \varkappa_{\alpha}\|z\|_{(\alpha)}{ }^{2}
$$

(Lemma 3 of $[11]$ ), since

$$
\|z\|_{(\alpha)}^{2}=\|z\|^{2}+\tau^{2}\left\|A_{\alpha} z\right\|^{2} \leqslant\left(1+\tau^{2}\left\|A_{\alpha}\right\|^{2}\right)\|z\|^{2} \leqslant \frac{1}{\delta_{\alpha}}\left(1+\tau^{2}\left\|A_{\alpha}\right\|^{2}\right)\left(A_{\alpha} z, z\right) .
$$

We now get

$$
\begin{aligned}
& \left(1+x_{1}\right)\|\bar{z}\|_{(1)}{ }^{2} \leqslant\left(1-x_{2}\right)\left\|z^{k}\right\|_{(2)}^{2}, \quad\left(1+x_{2}\right)\left\|z^{k+1}\right\|_{(2)}{ }^{2} \leqslant\left(1-x_{1}\right)\|\vec{z}\|_{(1)}{ }^{2}, \\
& \|\bar{z}\|_{(1)}{ }^{2} \leqslant \frac{1-x_{2}}{1+x_{1}}\left\|z^{k}\right\|_{(2)}{ }^{2}, \quad\left\|z^{k+1}\right\|_{(2)}{ }^{2} \leqslant \frac{1-x_{1}}{1+x_{2}}\|\vec{z}\|_{(1)}{ }^{2} \leqslant \rho_{1}^{2} \rho_{2}{ }^{2}\left\|z^{k}\right\|_{(2)}{ }^{2}
\end{aligned}
$$

From (14), $\left\|z^{v}\right\|_{(2)} \leqslant \rho^{v}\left\|z_{0}\right\|_{(2)}$. Let $\varepsilon>0$ be the required accuracy, so that $\left\|z^{v}\right\|_{(2)} \leqslant \varepsilon\left\|z_{0}\right\|_{(2)}$. It follows from this and the previous inequality that, to reduce the initial error in the ratio $1 / \varepsilon, v(\varepsilon)$ iterations are needed, where

$$
v(\varepsilon) \geqslant \ln \frac{1}{\varepsilon} \mid \ln \frac{1}{\rho}
$$

Corollary. Let $\delta=\min \left(\delta_{1}, \delta_{2}\right),\|A\|=\max \left(\left\|A_{1}\right\| .\left\|A_{2}\right\|\right)$. Then $\min \rho(\tau)=\rho\left(\tau^{*}\right)=\bar{\rho}, \quad \tau^{*}=1 /\|A\|$, where $\rho=(1-\eta) /(1+\eta)$, $\eta=\delta /\|A\|$.

2. We now assume the following conditions in addition to (13):

$\left\|A_{\alpha} y .\right\|^{2} \leqslant \Delta_{\alpha}\left(A_{\alpha} y, y\right), \quad \Delta_{\alpha}$ - const $>0, \quad \alpha-1,2, \quad y \in H$. (18) Lemma 1

For a linear positive $((A y, y) \geqslant 0)$ and selfadjoint operator $A$, 


$$
\|A y\|^{2} \leqslant\|A\|(A y, y), \quad y \in H .
$$

In fact, putting $z=A y$ in the generalized Cauch inequality: $(A y, z)^{2} \leqslant(A y, y)(A z, z)$, we get

$$
\begin{aligned}
\|A y\|^{4}=(A y, z)^{2} & \leqslant(A y, y)(A z, z) \leqslant\|A\|\|z\|^{2}(A y, y)= \\
& =\|A\|\|A y\|^{2}(A y, y) .
\end{aligned}
$$

Cancelling by $\|A y\|^{2}$, (19) follows.

Comparing (19) and (18), we see that, for a salfadjoint operator $A_{\alpha}$,

$$
\Delta_{\alpha}=\left\|A_{\alpha}\right\| \text {. }
$$

If $A_{\alpha}$ is any positive definite operator, then $\Delta_{\alpha} \leqslant\left\|A_{\alpha}\right\|^{2} / \delta_{\alpha}$, since

$$
\left\|A_{\alpha} y\right\|^{2} \leqslant\left\|A_{\alpha}\right\|^{2}\|y\|^{2} \leqslant\left\|A_{\alpha}\right\|^{2} \frac{1}{\delta_{\alpha}}\left(A_{\alpha} y, y\right) .
$$

Condition (18) is thus satisfied for any positive definite operator.

Theorem 2

Let $A_{1}, A_{2}$ satisfy conditions (13), (18). Formulae (14), (15) now hold, provided

$$
x_{\alpha}=\frac{2 \tau \delta_{\alpha}}{1+\tau^{2} \delta_{\alpha} \Delta_{\alpha}}, \quad \alpha=1,2
$$

It is sufficient to show that $2 \tau\left(A_{\alpha} z, z\right) \geqslant x_{a}\|z\|_{(\alpha)}{ }^{2}$, where $k_{\alpha}$ is given by (20). For,

$$
\|z\|(a)^{2}=\|z\|^{2}+\tau^{2}\left\|A_{\alpha} z\right\|^{2} \leqslant\|z\|^{2}+\tau^{2} \Delta_{\alpha}\left(A_{\alpha} z, z\right) \leqslant\left(\frac{1}{\delta_{\alpha}}+\tau^{2} \Delta_{\alpha}\right)\left(A_{\alpha} z, z\right)
$$

and

$$
\left(A_{\alpha} z, z\right) \geqslant \delta_{\alpha}\left(1+\tau^{2} \delta_{\alpha} \Delta_{\alpha}\right)^{-1}\|z\|_{(\alpha)}^{2}
$$

Theorem 3

Let conditions (13) and (18) be satisfied. Then the iterational process (2) with parameter $T$ equal to

$$
\tau=\tau^{*}=1 / \sqrt{\bar{\delta} \Delta}, \quad \delta=\min \left(\delta_{1}, \delta_{2}\right), \quad \Delta=\max \left(\Delta_{1}, \Delta_{2}\right),
$$


is convergent at the rate of the geometric progression with ratio

$$
\bar{\rho}=\frac{1-\sqrt{\eta}}{1+\sqrt{\eta}}, \quad \eta=\frac{8}{\Delta},
$$

so that

$$
\left\|y^{k-1}-v\right\|_{(2)} \leqslant \bar{\rho}^{k+1}\left\|y_{0}-v\right\|_{(2)} \text {. }
$$

To prove this theorem, we only need to observe that

$$
\begin{array}{lr}
\rho \leqslant \bar{\rho}=(1-\bar{x}) /(1+\bar{x}), & \bar{x}=2 \tau \delta /\left(1+\tau^{2} \delta \Delta\right), \\
\min \bar{\rho}(\tau)=\rho(\max \bar{x}), & \max \bar{x}=\bar{x}\left(\tau^{*}\right)=\sqrt{\eta} .
\end{array}
$$

Given a small characteristic parameter $\eta$, the numier of iterations $v(\varepsilon)$ satisfies

$$
v(8) \approx \frac{1}{\sqrt{\eta}} \ln \frac{1}{8}
$$

Notice that, given $\delta_{\alpha}, \Delta_{\alpha}, \alpha=1,2$, the value $\tau=\tau^{*}$ minimizing $p=\rho_{1} \rho_{2}$ can be found either graphically or by using tables of the functions $\rho^{2}(\tau)$.

It is clear from (14) that scheme (12) is convergent provided one of the operators, say $A_{1}$, is non-negative $\left(\delta_{1}=0\right)$, while the second is positive definite $\left(\delta_{2}>0\right)$; then

$$
\rho=\sqrt{\bar{\rho}}, \bar{\rho}=(1-\sqrt{\eta}) /(1+\sqrt{\eta}), \eta=\delta_{2} / \Delta_{2} \text { with } \tau=1 / \sqrt{\delta_{2} \Delta_{2}}
$$

3. We obtain bounds for the solution of the Cauchy problem(12) by another method.

In some cases convergence rate estimates can be improved. We shall assume that the parameter $\tau=\tau_{k}>0$ depends on $k$, so that

$$
\begin{gathered}
\left(E+\tau_{k+1} A_{1}\right) \bar{z}=\left(E-\tau_{k+1} A_{2}\right) z^{k}, \quad\left(E+\tau_{k+1} A_{2}\right) z^{k+1}=\left(E-\tau_{k+1} A_{1}\right) \bar{z} \\
k=0,1,2, \ldots, \\
z^{0}=z_{0} .
\end{gathered}
$$

We obtain from this [15]

$$
\begin{gathered}
z^{k+1}=T_{k+1} z^{k} \\
T_{k+1}=\left(E+\tau_{k+1} A_{2}\right)^{-1}\left(E-\tau_{k+1} A_{1}\right)\left(E+\tau_{k+1} A_{1}\right)^{-1}\left(E-\tau_{k+1} A_{2}\right) .
\end{gathered}
$$




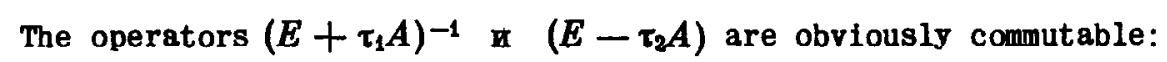

$\left(E+\tau_{1} A\right)^{-1}\left(E-\tau_{2} A\right)=\left(E-\tau_{2} A\right)\left(E+\tau_{1} A\right)^{-1}$. In fact,

$\left(E-\tau_{2} A\right)\left(E+\tau_{1} A\right)^{-1}=\left[\left(\frac{\tau_{2}}{\tau_{1}}+1\right) E-\frac{\tau_{2}}{\tau_{1}}\left(E+\tau_{1} A\right)\right]\left(E+\tau_{1} A\right)^{-1}=$

$$
=\left(\frac{\tau_{2}}{\tau_{1}}+1\right)\left(E+\tau_{1} A\right)^{-1}-\frac{\tau_{2}}{\tau_{1}}\left(E+\tau_{1} A\right)^{-1}\left(E+\tau_{1} A\right)=
$$

$=\left(E+\tau_{1} A\right)^{-1}\left[\left(\frac{\tau_{2}}{\tau_{1}}+1\right) E-\frac{\tau_{2}}{\tau_{1}}\left(E+\tau_{1} A\right)\right]=\left(E+\tau_{1} A\right)^{-1}\left(E-\tau_{2} A\right)$ $\left(\tau_{1}, \tau_{2}>0\right.$ arbitrary). Hence [15]

$$
\begin{gathered}
T_{k+1}=\left(E+\tau_{k+1} A_{2}\right)^{-1}\left(E+\tau_{k+1} A_{1}\right)^{-1}\left(E-\tau_{k+1} A_{1}\right)\left(E-\tau_{k+1} A_{2}\right), \\
\Sigma^{k+1}=\left(E+\tau_{k+1} A_{2}\right)^{-1} B_{1}\left(\tau_{k+1}, \tau_{k+1}\right) B_{2}\left(\tau_{k}, \tau_{k+1}\right) B_{1}\left(\tau_{k}, \tau_{k}\right) \ldots \\
\ldots B_{1}\left(\tau_{1}, \tau_{1}\right)\left(E-\tau_{1} A_{2}\right) z^{0}
\end{gathered}
$$

where

$$
B_{\alpha}\left(\tau_{m}, \tau_{n}\right)=\left(E+\tau_{m} A_{\alpha}\right)^{-1}\left(E-\tau_{n} A_{a}\right), \quad \alpha=1,2 .
$$

From (26)

$\left\|\left(E+\tau_{k+1} A_{2}\right) z^{k+1} \mathbb{K} \prod_{j=1}^{k+1}\right\| B_{1}\left(\tau_{j}, \tau_{j}\right)\left\|\prod_{j=1}^{k}\right\| B_{2}\left(\tau_{j}, \tau_{j+1}\right)\|\|\left(E-\tau_{1} A_{2}\right) z^{0} \|$.

We thus require a bound for the norm of the operator

$$
B\left(\tau_{1}, \tau_{2}\right)=\left(E+\tau_{1} A\right)^{-1}\left(E-\tau_{2} A\right)
$$

under the conditions

$$
\begin{array}{r}
(A y, y) \geqslant \delta\|y\|_{2}, \\
\|A y\|_{2} \leqslant \Delta(A, y) .
\end{array}
$$

This operator is the transition operator for the scheme

$$
\left(E+\tau_{1} A\right) y^{j+1}=\left(E-\tau_{2} A\right) y^{j} \text {. }
$$

Putting $T=T_{1}+T_{2}, T_{1}=\sigma, T_{2}=(1-\sigma) T$, where $\sigma=T_{1} / T$, we can rewrite the equation as

$$
\begin{gathered}
y_{\bar{t}}+A(\sigma y+(1-\sigma) \check{y})=0, \quad y_{\bar{t}}=\frac{y-\check{y}}{\tau}, \\
y=y^{j+1}, \quad \check{y}=y^{j}, \quad j=0,1,2, \ldots
\end{gathered}
$$


We utilize the following equations, which can be verified directly:

$$
\begin{gathered}
\sigma y+(1-\sigma)^{2} y=0.5(y+\check{y})+(\sigma-0.5) \tau y_{\bar{t}}, \\
\left(y_{\bar{t}}, \sigma \mathrm{y}+(1-\sigma) \check{y}\right)=0.5\left(\|y\|^{2}\right)_{\bar{t}}+(\sigma-0.5) \tau\left\|y_{\bar{t}}\right\|^{2}, \\
\|y\|^{2}+\|\check{y}\|^{2}=\frac{1}{2}\|y+\check{y}\|^{2}+\frac{1}{2} \tau^{2}\left\|y_{\bar{t}}^{\prime}\right\|^{2} .
\end{gathered}
$$

Lemma 2

We have

$$
\|\sigma y+(1-\sigma) \check{y}\|^{2}=\sigma\|y\|^{2}+(1-\sigma)\|\check{y}\|^{2}+\left(\sigma^{2}-\sigma\right) \tau^{2}\left\|y_{\bar{t}}\right\|^{2} .
$$

Using (33) and (35), we get

$$
\begin{gathered}
\|\sigma y+(1-\sigma) \check{y}\|^{2}=(\sigma y+(1-\sigma) \check{y}, \quad \sigma y+(1-\sigma) \check{y})=\frac{1}{4}\|y+\check{y}\|^{2}+ \\
+(\sigma-0.5) \tau\left(y+\check{y}, y_{\bar{t}}\right)+(\sigma-0.5) \tau^{2}\left\|y_{\bar{t}}\right\|^{2}=(\sigma-0.5)\left(\|y\|^{2}-\|\check{y}\|^{2}\right)+ \\
+\left(\sigma^{2}-\sigma\right) \tau^{2}\left\|y_{\bar{t}}\right\|^{2}+\frac{1}{2}\left(\frac{1}{2}\|y+\check{y}\|^{2}+\frac{1}{2} \tau^{2}\left\|y_{\bar{t}}\right\|^{2}\right)= \\
=(\sigma-0.5)\left(\|y\|^{2}-\|\check{y}\|^{2}\right)+\left(\sigma^{2}-\sigma\right) \tau^{2}\left\|y_{\bar{t}}\right\|^{2}+ \\
+0.5\left(\|y\|^{2}+\|\check{y}\|^{2}\right)=\sigma\|y\|^{2}+(1-\sigma)\|\check{y}\|^{2}+\left(\sigma^{2}-\sigma\right) \tau^{2}\left\|y_{\bar{t}}\right\|^{2} .
\end{gathered}
$$

We form the scalar product of (32) with $2 y^{(\sigma)}=2(\sigma y+(1-\sigma) y)$, and recall (30), (34) and (36)

$$
\begin{gathered}
\|y\|^{2}+2 \tau^{2}(\sigma-0.5)\left\|y_{\bar{t}}\right\|^{2}+2 \tau \delta L \sigma\|y\|^{2}+(1-\sigma)\|y\|^{2}+ \\
\left.+\left(\sigma^{2}-\sigma\right) \tau^{2}\left\|y_{\bar{t}}\right\|^{2}\right] \leqslant 0 .
\end{gathered}
$$

Hence follows the first energy inequality

$$
\begin{gathered}
(1+2 \sigma \tau \delta)\|y\|^{2}+2 \tau^{2} \omega\left\|y_{\bar{t}}\right\|^{2} \leqslant(1-2(1-\sigma) \tau \delta)\|\check{y}\|^{2}, \\
\|y\|^{2} \leqslant \frac{1-2(1-\sigma) \tau \delta}{1+2 \sigma \tau \delta}\|\check{y}\|^{2} \quad \text { for } \omega \geqslant 0,
\end{gathered}
$$

where

$$
\omega=\sigma-0.5-\tau \delta \sigma(1-\sigma) .
$$

Notice that $\omega \geqslant 0$ implies

$$
\sigma \geqslant \frac{1}{2 \tau \delta}\left[\tau \delta-1+\sqrt{1+\tau^{2} \delta^{2}}\right]=f(\tau \delta), \text { where } 0.5 \leqslant f(\tau \delta) \leqslant 1 .
$$


Now let $\omega<0$.

In this case we need a second energy inequality. We apply the operator $A^{-1}$ to (32)

$$
A^{-1} y_{\bar{t}}+\sigma y+(1-\sigma) \check{y}=0
$$

We form the scalar product of (40) with $2 \mathrm{~T}_{\bar{t}}$ and recall (34)

$$
2 \tau\left(A^{-1} y_{\bar{t}}, y_{\bar{t}}\right)+2(\sigma-0.5) \tau^{2}\left\|y_{\bar{t}}\right\|^{2}+\|y\|^{2}=\|\check{y}\|^{2} \text {. }
$$

Lemma 3

If $A$ satisfies $(30)$ and $\left(30^{\prime}\right)$, then

$$
\left(A^{-1} y, y\right) \geqslant \frac{1}{\Delta}\|y\|^{2}, \quad y \in H .
$$

For, putting $y=A v, v=A^{-1} y$ and using condition $\left(30^{\prime}\right)$, i.e. $\|A v\|^{2} \leqslant$ $\Delta(A v, v)$, we get

$$
\|y\|^{2}=\|A v\|^{2} \leqslant \Delta(A v, v)=\Delta\left(A^{-1} y, \zeta\right) .
$$

Using (42), we obtain from (41) the second energy inequality

$$
2 \tau\left(\frac{1}{\Delta}+(\sigma-0.5) \tau\right)\left\|y_{\bar{t}}\right\|^{2}+\|y\|^{2} \leqslant\|\underline{y}\|^{2} .
$$

Hence follows at once

Theorem 4

The difference scheme (32) is stable with respect to its initial data, so that $\left\|y^{j}\right\| \leqslant\left\|y^{0}\right\|$ with

$$
\sigma \geqslant 0.5-\frac{1}{\tau \Delta}
$$

We consider (37) with $\omega<0$ and (43) with

$$
\sigma>0.5-\frac{1}{\tau \Delta}
$$

It is easily observed that the condition $\omega<0$ and (44) are satisfied if 


$$
0.5-\frac{1}{\tau \Delta}<\sigma<\frac{\tau \delta-1+\sqrt{1+\tau^{2} \delta^{2}}}{2 \tau \delta} .
$$

We multiply (43) by $C_{T}$ and add the resulting inequality to (37)

$$
(1+2 \sigma \tau \delta+C \tau)\|y\|^{2} \leqslant(1-2(1-\sigma) \tau \delta+C \tau)\|\check{y}\|^{2},
$$

where

$$
C \tau=-\frac{\omega \tau \Delta}{1+(\sigma-0.5) \tau \Delta}=\frac{(\sigma-0.5) \tau \Delta+\sigma(1-\sigma) \tau^{2} 8 \Delta}{1+(\sigma-0.5) \tau \Delta} .
$$

After substituting (47) in (46), we get

$$
\|y\|^{2} \leqslant \frac{1-2(1-\sigma) \tau \delta+(1-\sigma)^{2} \tau^{2} \delta \Delta}{1+2 \sigma \tau \delta+\sigma^{2} \tau^{2} \delta \Delta}\|y\|^{2} .
$$

From this, and the equation $y=B\left(\tau_{1}, \tau_{2}\right) \check{y}=\left(E+\tau_{1} A\right)^{-1}\left(E-\tau_{2} A\right) \check{y}$ we get

$$
\left\|B\left(\tau_{1}, \tau_{2}\right)\right\| \leqslant \rho\left(\tau_{1}, \tau_{2}\right)=\sqrt{\frac{1-2 \tau_{2} \delta+\tau_{2}^{2} \delta \Delta}{1+2 \tau_{1} \delta+\tau_{1}^{2} \delta \Delta}}
$$

In particular, with $\tau_{1}=\tau_{2}=\tau$ we have

$$
\left\|(E+\tau A)^{-1}(E-\tau A)\right\| \leqslant \sqrt{\frac{1-x}{1+x}}=\rho(\tau), \quad x=\frac{2 \tau \delta}{1+\tau^{2} \delta \Delta} .
$$

The bound (49) is obtained under conditions (45) or with $\sigma>0.5$ $1 / \tau \Delta$ and $\omega=\sigma-0.5-\tau \delta \sigma(1-\sigma)<0$. It follows from this that

$$
\frac{\tau_{1}}{1+2 \tau_{1} \delta}<\tau_{2}<\tau_{1}+\frac{2}{\Delta} .
$$

We have now proved

Theorem 5

Let conditions $(30),\left(30^{\prime}\right)$ and $\left(5^{\prime}\right)$ be satisfied. Then

$$
\left\|\left(E+\tau_{1} A\right)^{-1}\left(E-\tau_{2} A\right)\right\| \leqslant \rho\left(\tau_{1}, \tau_{2}\right),
$$

where

$$
\rho\left(\tau_{1}, \tau_{2}\right)=\sqrt{\frac{1-2 \tau_{2} \delta+\tau_{2}^{2} \delta \Delta}{1+2 \tau_{1} \delta+\tau_{1}{ }^{2} \delta \Delta}}<1 .
$$


We now return to inequality (28). Recalling that

$$
\begin{gathered}
\left\|\left(E+\tau_{k+1} A_{2}\right) z^{k+1}\right\|^{2} \geqslant\left(1+x\left(\tau_{k+1}\right)\right)\left\|z^{k+1}\right\|^{2} \\
\left\|\left(E-\tau_{1} A_{2}\right) z^{0}\right\|^{2} \leqslant\left(1-x_{2}\left(\tau_{1}\right)\right)\left\|z^{0}\right\|_{(2)}^{2}, \quad\left\|z^{0}\right\|_{(2)}^{2}=\left\|z^{0}\right\|^{2}+\tau_{1}^{2}\left\|A_{2} z^{0}\right\|^{2}
\end{gathered}
$$

we get

$$
\left\|z^{(k+1)}\right\|_{(2)} \leqslant \prod_{j=1}^{k} \rho_{1}\left(\tau_{j}, \tau_{j}\right) \rho_{2}\left(\tau_{j}, \tau_{j+1}\right) \rho_{1}\left(\tau_{k+1}, \tau_{k+1}\right) \sqrt{\frac{1-x_{2}\left(\tau_{1}\right)}{1+x_{2}\left(\tau_{k+1}\right)}}\left\|z^{0}\right\|_{(2),}
$$

where $\left\|z^{k}\right\|_{(2)}=\left(\left\|z^{k}\right\|^{2}+\tau_{h}^{2}\left\|A_{2} z^{k}\right\|^{2}\right)^{1 / 2}, k=0,1,2, \ldots$.

With $\tau_{k}=\tau=$ const., this gives us

$$
\begin{gathered}
\left\|z^{k+1}\right\|_{(2)} \leqslant \rho^{k+1}\left\|z^{0}\right\|_{(2),}, \quad \rho=\sqrt{\rho_{1} \rho_{2}}, \\
\rho_{\alpha}^{2}=\frac{1-x_{\alpha}}{1+x_{\alpha}}, \quad x_{\alpha}=2 \tau \delta_{\alpha} /\left(1+\tau^{2} \delta_{\alpha} \Delta_{\alpha}\right), \quad \alpha=1,2,
\end{gathered}
$$

which we obtained earlier (Theorem 2).

Noticing that $\left(1+\tau^{2} \delta_{2}^{2}\right)\|z\|^{2} \leqslant\|z\|_{(2)}^{2} \leqslant\left(1+\tau^{2}\left\|A_{2}\right\|^{2}\right)\|z\|^{2}$; we obtain from (55)

$$
\left\|z^{k+1}\right\| \leqslant \sqrt{\frac{1+\tau^{2}\left\|A_{2}\right\|^{2}}{1+\tau^{2} \delta_{2}^{2}}} \rho^{k+1}\left\|z^{0}\right\| .
$$

The bound (54) shows that iterations in accordance with scheme (2) are convergent for $\tau_{k} \geqslant \tau_{k-1}$, or $\tau_{k} \leqslant \tau_{k-1}$, provided that $\tau_{k-1}(1+$ $\left.2 \tau_{k-1} \delta\right)^{-1}<\tau_{k}<\tau_{k-1}+2 / \Delta$. But the fastest convergence can be obtained for $\tau_{k}=\tau_{k-1}=\tau$ by a suitable choice of $T$.

Note. An alternative, equivalent statement of Theorem 5 is: if conditions (30), $\left(30^{\prime}\right)$ and (45) are satisfied, then

$$
\left\|(E+\sigma \tau A)^{-1}(E-(1-\sigma) \tau A)\right\| \leqslant \rho, \quad \rho=\left(\frac{1-2(1-\sigma) \tau \delta+(1-\sigma)^{2} \tau^{2} \delta \Delta}{1+2 \sigma \tau \delta+\sigma^{2} \tau^{2} \delta \Delta}\right)^{1 / 2}
$$

4. We consider some special cases.

Let $H=H_{N}$ be an $N$-dimensional space, and $A$ a linear positive definite, selfadjoint operator in $H_{N}$. Then have the familiar limit $\left\|(E+\tau A)^{-1}(E-\tau A)\right\| \leqslant \max \left(\frac{1-\tau \lambda_{1}}{1+\tau \lambda_{1}}, \frac{\tau \lambda_{N}-1}{\tau \lambda_{N}+1}\right)$ for $\frac{1}{\lambda_{N}} \leqslant \tau \leqslant \frac{1}{\lambda_{1}}$, 
where $\lambda_{1}=\delta$ and $\lambda_{N}=\|A\|$ are the minimum and maximum eigenvalues respectively of $A$. For, let $A \xi_{s}=\lambda_{s} \xi_{s}, s=1,2, \ldots, N$, where $\left\{\lambda_{s}, \xi_{s}\right\}$ is the system of eigenvalues and eigenfunctions of $A$, and $A, \check{y}=\sum_{s=1}^{N} c_{s} \xi_{s}$. Then

$$
\begin{gathered}
y=(E+\tau A)^{-1}(E-\tau A) \check{y}=\sum_{s=1}^{N} \frac{1-\tau \lambda_{s}}{1+\tau \lambda_{s}} c_{s} \xi_{s} \\
\|y\| \leqslant \max _{1 \leqslant s \leqslant N}\left|\frac{1-\tau \lambda_{s}}{1+\tau \lambda_{s}}\right| \leqslant \max \left(\frac{1-\tau \lambda_{1}}{1+\tau \lambda_{1}}, \frac{\tau \lambda_{N}-1}{\tau \lambda_{N}+1}\right)
\end{gathered}
$$

for $\frac{1}{\lambda_{N}} \leqslant \tau \leqslant \frac{1}{\lambda_{1}}$.

Taking $\tau^{*}=1 / \sqrt{\lambda_{1} \lambda_{N}}$, we get

$$
\left\|\left(E+\tau^{*} A\right)^{-1}\left(E-\tau^{*} A\right)\right\| \leqslant \rho^{*}, \quad \rho^{*}=\frac{1-\sqrt{\eta}}{1+\sqrt{\eta}}, \quad \eta=\frac{\lambda_{1}}{\lambda_{N}} .
$$

In this case, Theorem 5 gives us

$$
\left\|\left(E+\tau^{*} A\right)^{-1}\left(E-\tau^{*} A\right)\right\| \leqslant \sqrt{\rho^{*}}
$$

with the same value $\tau=\tau^{*}, \lambda_{1}=\delta, \Delta=\|A\|=\lambda_{N}$. Let $A_{1}, A_{2}$ be commutable. Now, (26) gives us

$$
z^{k+1}=\prod_{j=1}^{k+1} B_{1}\left(\tau_{j}, \tau_{j}\right) B_{2}\left(\tau_{j}, \tau_{j}\right) z^{0}
$$

If $H=H_{N}$ and at least the operator $A_{2}$ is selfadjoint, we have to use a cyclical system of parameters $\tau_{1} \geqslant \tau_{2} \geqslant \ldots \geqslant \tau_{k_{0}}, T_{k} t_{n k_{0}}=\tau_{k}$ with $1 \leqslant k \leqslant k_{0}, n=1,2, \ldots, n_{0}$. Let $A_{1}$ be positive. We can now employ a one-dimensional version of the $\left\{\tau_{k}\right\}$. We obtain for $v(\varepsilon)$

$$
v(\varepsilon) \approx \ln \frac{1}{8} / \ln \frac{1}{\eta_{2}}, \quad \eta_{2}=\frac{\lambda_{1}^{(2)}}{\lambda_{N}^{(2)}},
$$

where $\lambda_{1}^{(2)}$ and $\lambda_{N}^{(2)}$ are the minimum and maximum eigenvalues of $A_{2}$.

The case when $H=H_{N}, A_{1}^{*}=A_{1}, A_{2}^{*}=A_{2}$ and $A_{1} A_{2}=A_{2} A_{1}$ has been fully investigated $[17,18]$, and we shall not dwell on $i t$. 
A theorem follows from the above that we shall utilize in Section 3 . paragraph 3 .

Theorem 6

Let $H=H_{N}, A_{1}$ and $A_{2}$ be positive - definite operators, and $A_{2}$ selfadjoint. The following limit now holds for the solution of problem (12):

$$
\left\|z^{k+1}\right\|_{(2)} \leqslant \rho\left\|z^{k}\right\|_{(2)} \text { for } \tau=\tau^{*}=\frac{1}{\sqrt{\lambda_{1}^{(2)} \lambda_{N}^{(2)}}},
$$

where $\|z\|_{(2)}^{2}=\|z\|^{2}+\left(\tau^{*}\right)^{2}\left\|A_{2} z\right\|^{2}, \quad \rho=\rho_{1} \rho_{2}^{*}, \quad \rho_{2}^{*}=\left(1-\sqrt{\eta_{2}}\right) /\left(1+\sqrt{\eta_{2}}\right)$, $\rho_{1}=\sqrt{\left(1-x_{1}^{*}\right) /\left(1+x_{1}^{*}\right)}, \quad x_{1}^{*}=2 \tau^{*} \delta_{1} /\left(1+\left(\tau^{*}\right)^{2} \delta_{1} \Delta_{1}\right)$.

\section{Examples}

We consider some examples of the application of the limits obtained in section 2. We use a standard method of investigation: we construct the mesh, then introduce the scalar product on the set of mesh functions and determine the operators $A_{1}, A_{2}$; usfing the difference analogues of Green's formulae and other simple devices, we then find the characteristic parameters $\delta_{\alpha}, \Delta_{\alpha}$. After this, we apply Theorem 2 or Theorem 6 (if e.g. $A_{2}$ is selfadjoint).

1. The Dirichlet difference problem for the selfconjugate elliptic equation in an arbitrary region.

We consider the Dirichlet problem

$$
\begin{gathered}
\left(L_{1}+L_{2}\right) u=-f(x), \quad x=\left(x_{1}, x_{2}\right) \in G,\left.\quad u\right|_{\Gamma}=\mu_{(}(x) \\
h_{\alpha} u=\frac{\partial}{\partial x_{\alpha}}\left(k_{\alpha}(x) \frac{\partial u}{\partial x_{\alpha}}\right), \quad 0<c_{1} \leqslant k_{\alpha}(x) \leqslant c_{2}, \quad \alpha=1,2 \\
c_{1}, c_{2}=\text { const }>0 .
\end{gathered}
$$

in an arbitrary region $G+\Gamma$ of the $\left(x_{1}, x_{2}\right)$ plane.

We construct in $\bar{G}=G+\Gamma$ the mesh $\bar{\omega}_{h, q}=\omega_{h, q}+\gamma_{h}$ by dividing the plane by the straight lines $x_{\alpha}=i_{\alpha} h_{\alpha}$, where the interval $h_{\alpha}$ in $x_{\alpha}$ is uniform. 
The mesh boundary $\gamma_{h}$ consists of the points of intersection of the straight lines $x_{\alpha}=i_{\alpha} h_{\alpha}$ with the boundary $\Gamma$ of region $G$. We impose the condition

$$
h_{\alpha}^{*} \geqslant q h_{\alpha}
$$

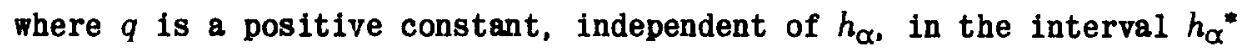
close to the boundary $\Gamma$. Such a mesh can always be obtained e.g. by discarding the base-points at which condition (62) is violated (we assume as always that the set of base-points of this mesh $\omega_{h, q}$ is connected). It is in general non-uniform close to the boundary.

We write the second order difference scheme for problem (61)

$$
\left(\Lambda_{1}+\Lambda_{2}\right) v=-f(x), \quad x \in \omega_{h, q},\left.\quad v\right|_{v_{h}}=\mu(x) .
$$

Here, $\Lambda_{\alpha} v=\left(a_{\alpha}(x) v_{\bar{x}_{\alpha}}\right) \hat{x}_{\alpha}, \quad a=1,2, \quad 0<c_{1} \leqslant a_{\alpha}(x) \leqslant c_{2}$ (see [19] for the notation).

This system of equations is solved by means of ADI iterations

$$
\begin{gathered}
\bar{y}-\tau \Lambda_{1} \bar{y}=y^{k}+\tau \Lambda_{2} y^{k}+\tau f, \quad k=0,1,2 \ldots, \\
y^{h+1}-\tau \Lambda_{2} y^{k+1}=\bar{y}+\tau \Lambda_{1} \bar{y}+\tau f, \quad x \in \omega_{h, q}, \\
\bar{y}=\mu(x), \quad y^{k+1}=\mu(x) \quad \text { for } x \in \gamma_{n} .
\end{gathered}
$$

We obtain problem (12) for the error $z^{k+1}=y^{k+1}-v A_{\alpha}=-\Lambda_{\alpha}, \alpha=1,2$, are operators in the space $\Omega$ of mesh functions specified at $\omega_{h, q}$ and vanishing on the boundary $\gamma_{h}$. The scalar product in $Q$ is

$$
(y, v)=\sum_{\omega_{h, q}} y(x) v(x) H, \quad H=\hbar_{1} \hbar_{2}, \quad \hbar_{\alpha}=0.5\left(h_{\alpha}+h_{\alpha}^{\left(+1_{\alpha}\right)}\right) .
$$

To utilize Theorems 1 and 2 , we have to prove that the $A_{\alpha}$ are positive definite and selfadjoint. Green's difference formulae give

$$
\left(A_{\alpha} y, y\right) \geqslant \delta_{\alpha}\|y\|^{2}, \quad \delta_{\alpha}=\frac{4 c_{1}}{D_{\alpha}^{2}}, \quad\left(A_{\alpha} y, v\right)=\left(y, A_{\alpha} v\right) .
$$

Further, we obtain

$$
\left\|A_{\alpha}\right\| \leqslant \frac{4 c_{2}}{q h_{\alpha_{a}}^{2}}=\Delta_{\alpha}, \quad \alpha=1,2 \text {, so that } \eta_{\alpha}=\frac{\delta_{\alpha}}{\Delta_{\alpha}}=\frac{c_{1} g h_{\alpha}^{2}}{c_{a} D_{a}^{2}} .
$$

Here $D_{\alpha}$ is the diameter of region $G$ in the $x_{\alpha}$ direction. By Theorem 6, the convergence of the ADI scheme (64) follows at once from (65), (66). The parameter $T$ is obtained from the minimization condition for 


$$
\rho=\max \left(\left|\frac{1-\tau \delta_{1}}{1+\tau \delta_{1}}\right|,\left|\frac{1-\tau \Delta_{1}}{1+\tau \Delta_{1}}\right|\right) \max \left(\left|\frac{1-\tau \delta_{2}}{1+\tau \delta_{2}}\right|,\left|\frac{1-\tau \Delta_{2}}{1+\tau \Delta_{2}}\right|\right)
$$

A cruder method of fixing $T^{*}$ is as follows. Let $h=\min \left(h_{1}, h_{2}\right), D=$ $\max \left(D_{1}, D_{2}\right), \delta=\min \left(\delta_{1}, \delta_{2}\right)=4 c_{1} / D^{2}, \Delta=4 c_{2} / q h^{2}, \Delta_{1} \leqslant \Delta, \Delta_{2} \leqslant \Delta$. We now get from (67)

$$
\begin{gathered}
\rho \leqslant \bar{\rho}=\left(\frac{1-\sqrt{\tilde{q}} h / D}{1+\sqrt{\tilde{q}} h / D}\right)^{2} \text { with } \tau^{*}=\frac{1}{4 \sqrt{c_{1} c_{2}}} h D \sqrt{q}, \\
\tilde{q}=q \frac{c_{1}}{c_{2}},
\end{gathered}
$$

while for $\nu(E)$ we have*

$$
v(8) \approx \sqrt{\frac{c_{2}}{c_{1}}} \frac{D}{h \sqrt{q}} \ln \frac{1}{8} .
$$

A formula $\nu(\varepsilon)=O(1 / h$ ln $1 / \varepsilon)$ is also obtained when $\omega_{h}$ is any nonuniform mesh whose intervals $h_{\alpha}\left(x_{i}\right)$ satisfy at all base-points $x_{i} \in \bar{\omega}_{h}$ the condition $h_{\alpha}\left(x_{i}\right) \geqslant h$, where $h=$ const. $>0$. Here, $\Lambda_{\alpha} v=\left(a_{\alpha} \bar{x}_{\alpha}\right)^{\prime} \hat{x}_{\alpha}$ everywhere.

2. We now consider the Dirichlet problem for a non-selfadjoint elliptic operator with constant coefficients

$$
\left(\widetilde{L}_{1}+\widetilde{L}_{2}\right) u=-f(x), \quad x \in G,\left.\quad u\right|_{\Gamma}=\mu(x), \quad \widetilde{L}_{\alpha} u=\frac{\partial^{2} u}{\partial x_{\alpha}^{2}}+r_{\alpha} \frac{\partial u}{\partial x_{\alpha}} \cdot
$$

To solve this on the mesh $\omega_{h, q}$, we use a monotonic scheme of the second order of accuracy [20]

$$
\begin{gathered}
\left(\widetilde{\Lambda}_{1}+\widetilde{\Lambda}_{a}\right) v=-f(x), \quad x \in \omega_{h, q}, \quad v=\mu(x) \text { for } x \in \gamma_{h}, \\
\tilde{\Lambda}_{\alpha} v=v_{\alpha} v_{\tilde{x}_{\alpha} \hat{x}_{\alpha}}+b_{\alpha}{ }^{+} v_{\hat{x}_{\alpha}}+b_{\alpha}^{-} v_{\hat{x}_{\alpha}}, \\
b_{\alpha}{ }^{+}=\frac{r_{\alpha}+\left|r_{\alpha}\right|}{2} \geqslant 0, \quad b_{\alpha}^{-}=\frac{r_{\alpha}-\left|r_{\alpha}\right|}{2} \leqslant 0,
\end{gathered}
$$

- N.S. Bakhvalov kindly informed me that he previously obtained formula (69) for boundary conditions without drift (scheme $O(h)$ ) and $k_{\alpha}=$ const. 


$$
v_{\alpha}=1-\frac{R_{a}}{1+R}, \quad R_{a}=0.5 h_{\alpha}\left|r_{a}\right|, \quad a=1,2 ; R=R_{1}+R_{2}
$$

The operators $\tilde{A}_{\alpha}=-\tilde{\Lambda}_{\alpha}$, defined on $\Omega$ are positive - definite

$$
\left(\widetilde{A}_{\alpha^{z}}, z\right) \geqslant \frac{4}{D_{\alpha}^{2}}\|z\|^{2}, \quad z \in \Omega, \quad \delta_{\alpha}=\frac{4}{D_{\alpha^{2}}^{2}},
$$

and they satisfy

$$
\begin{array}{cl}
\left\|\tilde{A}_{\alpha} z\right\|^{2} \leqslant \frac{4\left(1+c_{3} h_{\alpha}\right)}{q h_{\alpha}{ }^{2}}\left(\widetilde{A}_{\alpha} z, z\right), & c_{3}=\max \left(\left|r_{1}\right|,\left|r_{2}\right|\right), \\
\Delta_{\alpha}=\frac{4\left(1+c_{3} h_{\alpha}\right)}{q h_{\alpha}{ }^{2}}, & \alpha=1,2 .
\end{array}
$$

Property (72) can be proved by using Green's first difference formula [19]

$\left(\tilde{A}_{\alpha} z, z\right)=\left(-\widetilde{\Lambda}_{\alpha} z, z\right)=v_{\alpha}\left(1, z_{x_{\alpha}}^{2}\right]_{\alpha}+\frac{1}{2}\left(b_{\alpha}^{+}-b_{\alpha}{ }^{-}\right) h_{\alpha}\left(1, z_{x_{\alpha}}^{2 i}\right]_{\alpha}=$ $=\left(v_{\alpha}+R_{\alpha}\right)\left(1, z_{\bar{x}_{\alpha}}^{2}\right]_{\alpha} \geqslant\left(1-\frac{R_{a}}{1+R}+R_{\alpha}\right) \frac{4}{D_{\alpha}^{2}}\|z\|^{2} \geqslant \frac{4}{D_{\alpha}^{2}}\|z\|^{2}, \quad z \in \Omega$, since $\|z\|^{2} \leqslant \frac{D_{\alpha}^{2}}{4}\left\|z_{\bar{x}_{\alpha}}\right\| \alpha^{2}$ (for notation see [19]).

We prove (73) by means of the triangle inequality

$$
\begin{aligned}
& \left\|\widetilde{A}_{\alpha} z\right\| \leqslant v_{\alpha}\left\|z_{\bar{x}_{\alpha} \hat{x}_{\alpha}}\right\|+b_{\alpha}^{+}\left\|z_{\hat{x}_{\alpha}}\right\|+\left|b_{\alpha}{ }^{-}\right|\left\|z_{\bar{x}_{\alpha}}\right\| \leqslant v_{\alpha} \frac{2}{\sqrt{q} h_{\alpha}}\left(1, z_{\bar{x}_{\alpha}}^{2}\right]_{\alpha}^{1 / 2}+ \\
& +\frac{\left|r_{\alpha}\right|}{\sqrt{q}}\left(1, z_{x_{\alpha}}^{2}\right]_{\alpha}^{1 / a}=\frac{1}{\sqrt{q}}\left(\frac{2 v_{\alpha}}{h_{\alpha}}+\left|r_{\alpha}\right|\right)\left(1, z_{x_{\alpha}}^{2}\right]_{\alpha}^{1 / \alpha}=\frac{2\left(v_{\alpha}+R_{\alpha}\right)}{\sqrt{q} h_{a}}\left(1, z_{x_{\alpha}}^{2} j\right]_{\alpha}^{1 / 4} .
\end{aligned}
$$

If we now recall the identity $\left(A_{\alpha} z, z\right)=\left(v_{\alpha}+R_{\alpha_{\alpha}}\right)\left(1, z_{x_{\alpha}}^{2}\right]_{\alpha}$, we get

$$
\left\|A_{\alpha} z\right\|^{2} \leqslant \frac{4}{q h_{\alpha}^{2}}\left(v_{\alpha}+R_{\alpha}\right)\left(A_{\alpha} z, z\right) .
$$

From (72), (73), the ADI scheme for (71) is convergent for any $T>0$. If $h_{1}=h_{2}=h, D=\max \left(D_{1}, D_{2}\right)$, then 


$$
\rho \leqslant \bar{\rho}=\frac{1-\sqrt{\eta}}{1+\sqrt{\eta}}, \quad \eta=\frac{q h^{2}}{\left(1+\epsilon_{3} h\right) D^{2}} \text { with } \tau=\tau^{*}=\frac{1}{\sqrt{8 \Delta}}
$$

where $\delta=4 / D^{2}, \Delta=4\left(1+c_{3} h\right) / q h^{2}$. The number of iterations obeys the asymptotic formula

$$
v(\varepsilon)=\ln \frac{1}{\varepsilon} / \ln \frac{1}{\rho} \approx \frac{D}{\sqrt{q} h} \ln \frac{1}{\varepsilon} .
$$

A similar formula can be obtained for the Dirichlet difference problem in the case of the non-selfadjoint elliptic operator with variable coefficients

$$
\begin{aligned}
\sum_{\alpha=1}^{2}\left[\frac{\partial}{\partial x_{\alpha}}\left(k_{\alpha}(x) \frac{\partial u}{\partial x_{\alpha}}\right)+r_{\alpha}(x) \frac{\partial u}{\partial x_{\alpha}}\right]-q_{0}(x) u=-f(x), \\
q_{0}(x) \geqslant c_{1}{ }^{\prime}>0 .
\end{aligned}
$$

However, extra restrictions have to be imposed here on the coefficients and the mesh interval.

3. As our third example illustrating the application of the general theory of Section 2, we consider iterational schemes for solving the integro-differential equation for radiation transport in a layer

$$
\begin{gathered}
\mu \frac{\partial \varphi}{\partial x}+\sigma \varphi=\frac{1}{2} \sigma_{s} \int_{-1}^{1} \varphi\left(x, \mu^{\prime}\right) d \mu^{\prime}+f(x, \mu), \quad 0 \leqslant x \leqslant H, \quad-1 \leqslant \mu \leqslant 1 \\
\varphi(0, \mu)=\xi(\mu), \quad \mu>0 ; \quad \varphi(H, \mu)=\eta(\mu), \quad \mu<0 \\
\left(\sigma>0, \sigma>\sigma_{s}>0\right) .
\end{gathered}
$$

This problem was considered in $[12,13]$. Following $[13]$, we introduce the mesh $\omega_{h l}=\left\{\left(x_{i}=i h, \mu_{j}=j l\right), i=0,1, \ldots, n,-1 \leqslant j \leqslant m, j \neq 0\right.$, $h=H / n, l=1 / m\}$ and approximate the operators

$$
L_{1} \varphi=\mu \partial \varphi / \partial x, \quad L_{2} \varphi=\sigma \varphi-\frac{1}{2} \sigma_{s} \int_{-1}^{1} \varphi\left(x, \mu^{\prime}\right) d \mu^{\prime}
$$

by means of operators $\Lambda_{1}, \Lambda_{2}$ :

$$
\Lambda_{1} v=\left\{\begin{array}{ll}
\mu v_{\bar{x}}, & \mu>0, \\
\mu v_{x}, & \mu<0 ;
\end{array} \quad \Lambda_{2} v=\left(\Lambda_{2} v\right)_{i j}=\sigma v_{i j}-\frac{1}{2} \sigma_{s} \sum_{\substack{v=-m \\
v \neq 0}}^{m} c_{v} v_{i v},\right.
$$


where $\left(v_{\bar{x}}\right)_{i j}=\left(v_{i j}-v_{i-1, j}\right) / h, \quad\left(v_{x}\right)_{i j}=\left(v_{i+1, j}-v_{i j}\right) / h$.

We associate problem (76) - (77) with the equation $\Lambda_{1} v+\Lambda_{2} v=f$ with boundary conditions (77). Let $\Omega$ be the space of functions specified on the mesh $\omega_{h l}$ and vanishing on its boundary, i.e. with $i=0,1 \leqslant j \leqslant m$ and $i=n,-m \leqslant j \leqslant-1$. We introduce the operators $A_{1} v=\Lambda_{1} v, A_{2} v=$ $\Lambda_{2} v$ with $v \in \Omega$. The difference scheme for problem (76) - (77) can be written as

$$
\left(A_{1}+A_{2}\right) v=f, \quad v \in \Omega
$$

(the right-hand side $\tilde{f}$ takes account of the boundary conditions).

The scalar product

$$
(z, v)=(z, v)^{-}+(z, v)^{+} \text {and the norm }\|z\|=\sqrt{(z, z),}
$$

can be introduced naturally into space $\Omega$, where

$$
(z, v)^{-}=\sum_{i=0}^{n-1} \sum_{j=-m}^{-1} z_{i j} v_{i j} h l, \quad(z, v)^{+}=\sum_{i=1}^{n} \sum_{j=1}^{m} z_{i j} v_{i j} h l .
$$

The operators $A_{1}, A_{2}$ are positive definite in $\Omega$. If the $c_{v}$ are fixed in accordance with [13] (e.g. $c_{v}=l$ ), then $A_{2}$ is selfadjoint.

It can easily be shown that

$$
\begin{gathered}
\left(A_{1} z, z\right) \geqslant \delta_{1}\|z\|^{2}, \quad\left\|A_{1} z\right\|^{2} \leqslant \Delta_{1}\left(A_{1} z, z\right), \quad \delta_{1}=\frac{h l}{H^{2}}, \quad \Delta=\frac{2}{h},(80) \\
\sigma_{c}\|z\|^{2} \leqslant\left(A_{2} z, z\right) \leqslant \sigma\|z\|^{2}, \quad \sigma_{c}=\sigma-\sigma_{s}>0 .
\end{gathered}
$$

The bounds (81) are obtained in [13]. We prove (80). Using the identities $z z=0.5\left(z^{2}\right)_{\bar{x}}=0.5 h z^{2}, z z_{x}=0.5\left(z^{2}\right)_{x}-0.5 h z_{x}^{2}$ and the inequalities

$$
z_{1}^{2} \leqslant i h \sum_{k=1}^{n} h z_{\bar{x}, k}^{2}, \quad \sum_{i=1}^{n-1} z_{i}^{2} h \leqslant \frac{H(H-h)}{2} \sum_{k=1}^{n} h z_{\bar{x}, k}^{2},
$$

we get

$$
\left(\mu z_{\bar{x}}, z\right)^{+}=0.5 \sum_{j=1}^{m} \mu_{j}\left(z_{n}^{j}\right)^{2} l+0.5 h\left(\mu, z_{\bar{x}}^{2}\right)^{+} \geqslant \frac{h}{H^{2}}\left(\mu, z^{2}\right)^{+} \geqslant \frac{h l}{H^{2}}\left(1, z^{2}\right)^{+}
$$


and similarly

$$
\left(\mu z_{x}, z\right)^{-}=-0.5 \sum_{j=-m}^{-1} \mu_{j}\left(z_{0}^{j}\right)^{2} l-0.5 h\left(\mu, z_{x}^{2}\right)^{-} \geqslant \frac{h l}{H^{2}}\left(1, z^{2}\right)^{-}
$$

Combining (82) and (83), we get $\left(A_{1} z, z\right) \geqslant \delta_{1}\|z\|^{2}$. If we recall that $\left(A_{1} z, z\right) \geqslant 0.5 h\left[\left(\mu, z_{x}^{2}\right)^{+}-\left(\mu, z_{x}^{2}\right)^{-}\right]$, we obtain at once

$$
\begin{aligned}
\left\|A_{1} z\right\|^{2}= & \left(\mu^{2}, z_{\bar{x}}^{2}\right)^{+}+\left(\mu^{2}, z_{x}^{2}\right)^{-} \leqslant \\
& \leqslant\left(\mu, z_{\bar{x}}^{2}\right)^{+}+\left(|\mu|, z_{x}^{2}\right)^{-} \leqslant \frac{\left(A_{1} z, z\right)}{0.5 h}=\Delta_{1}\left(A_{1} z, z\right), \quad \Delta_{1}=\frac{2}{h} .
\end{aligned}
$$

The scheme (5) with $f_{1}=0, f_{2}=0$ is used in [13] to solve problem (78). As shown in Section 1, this scheme is algebraically identical with the ADI scheme (2). Its convergence for any $\tau$ therefore follows from [11]. We estimate its rate of convergence. The estimate of [11] is too crude, since it takes no account of the fact that $A_{2}$ is selfadjoint. Theorem 5 gives

$$
\left\|z^{v}\right\|_{(2)} \leqslant \rho_{v}\left\|z_{0}\right\|_{(2)}, \quad\|z\|_{(2)}^{2}=\|z\|^{2}+\tau^{2}\left\|A_{2} z\right\|^{2},
$$

where

$$
\begin{gathered}
\rho=\rho_{1} \rho_{2}, \quad \rho_{1}=\sqrt{\frac{1-x_{1}}{1+x_{1}}}, \quad x_{1}=2 \delta_{1} \tau, \quad \delta_{1} \Delta_{1}=\frac{2 l}{H^{2}}, \\
\rho_{2}=\frac{1-\sqrt{\eta_{2}}}{1+\sqrt{\eta_{2}}}, \quad \eta_{2}=\frac{\sigma_{c}}{\sigma} \text { with } \tau=\frac{1}{\sqrt{\sigma \sigma_{c}}} .
\end{gathered}
$$

Hence it follows that

$$
v(\varepsilon)=\ln \frac{1}{\varepsilon} / \ln \left(\rho_{1}\left(1-\sqrt{\eta_{2}}\right)\left(1+\sqrt{\eta_{2}}\right)^{-1}\right) .
$$

By Theorem 2, the ADI scheme is also convergent with $\sigma_{c}=0$. In this case $\rho=\rho_{1}=\left(\left(1-\overline{\eta_{1}}\right) i\left(1+\sqrt{\eta_{1}}\right)\right)^{1 / 2}, \eta_{1}=h^{2} l / 2 H^{2}$, so that, as $h \rightarrow 0, l \rightarrow 0$ we have $v(\varepsilon) \approx H / h \sqrt{l}$. For comparison, we mention that the special methods used in [13] only enabled a bound to be obtained for the spectral radius $p(T)$ of the non-selfadjoint transition operator

$$
\begin{gathered}
T=\left(E+\tau A_{2}\right)^{-1}\left(E+\tau A_{1}\right)^{-1}\left(E-\tau A_{1}\right)\left(E-\tau A_{2}\right), \\
\rho(T) \leqslant \frac{1-\sqrt{\eta_{2}}}{1+\sqrt{\eta_{2}}} .
\end{gathered}
$$


The economy of a method is characterized by the amount of computational work needed to obtain a solution to a given accuracy.

From this point of view, it is interesting to compare the scineme of [13] with the simple iterational scieme

$$
\begin{gathered}
\left(\Lambda_{1}+\sigma E\right) y^{k+1 / 2}=\widetilde{\Lambda}_{2} y^{k}+f, \quad\left(\tilde{\Lambda}_{2} y^{k}\right)_{i j}=\frac{\sigma_{s}}{2} \sum_{\substack{\nu=-m \\
\nu \neq 0}}^{m} c_{v} y_{i v}{ }^{m}, \\
\left(\Lambda_{1}+\sigma E\right) y^{k+1}=\widetilde{\Lambda}_{2} y^{k+1 / 2}+f .
\end{gathered}
$$

Ising the fact that the maximun principle applies for $\Lambda_{1}+\sigma t$, we can easily show that scneine (85) is convergent in a uniform metric with ratio $\rho_{0}=\left(\sigma_{s} / \sigma\right)^{2}$, so that

$$
\left\|y^{k+1}-v\right\|_{0} \leqslant \rho_{0}\left\|y^{k}-v\right\|_{0}, \quad\|y\|_{0}=\max _{i, j}\left|y_{i j}\right|, \quad \rho_{0}=\left(\sigma_{s} / \sigma\right)^{2}
$$

Following [13], we take as the characteristic convergence rate of the scheme of $[13]$ the ratio $\left.\rho^{*}=\left(1-\sqrt{\eta_{2}}\right) / 1+\sqrt{\eta_{2}}\right), \quad \eta_{2}=\sigma_{c} / \sigma=1-$ $\sigma_{s} / \sigma$. Comparing $\rho^{*}$ and $\rho_{0}$, we see that there exists a region of variation of the parameter $\eta=\left(\sigma_{c} / \sigma\right)$ with $4 \eta^{3}+4 \eta-\eta^{2}>4$, in which $\rho_{0}<\rho^{*}$.

of course it would be premature to draw any înal conclusion regarding the advantage of one method over another from majorant bounds. However, our comparison shows that further investigation is needed (experimental as well as theoretical) into whether the ADI scheme is worth while for the transport equation. We need a more precise concept of the economy of a scheme for this equation. In any case, the "economy of a scheme of the first order in $T$ " discussed in section 5 of [13] is not economy in the sense of minimizing the computational work: in this case, the formula for the number of iterations is $v(\varepsilon)=(1 / \varepsilon) \ln (1 / \varepsilon)$, since $t=O(\varepsilon)$.

4. Theorems 2 and 6 offer an estimate of the convergence of the ADI scheme (12) of Section 1 and enable optimal (more precisely, p-minimizing) values to be obtained for the relaxation parameter $T$ for many other problems.

Decomposition of the operator $A$ of the initial stationary problem into a sum of two positive definite operators $A_{1}, A_{2}$ can be performed in various ways. We illustrate this by the example of the Dirichlet difference problem for the Poisson equation 


$$
\Delta u=\sum_{a=1}^{p} \frac{\partial^{2} u}{\partial x_{\alpha^{2}}}=-t
$$

in an arbitrary $p$-dimensional region $G=G+\Gamma$ on the mesh $\omega_{h, q}$ (see paragraph 1, Section 3$)$. We write the operator

$$
\Lambda y=-\sum_{a=1}^{p} y_{\bar{x}_{a} \hat{x}_{a}}
$$

as the sum $\Lambda=\Lambda^{-}+\Lambda^{+}$, where

$$
\Lambda^{-} y=-\sum_{\alpha=1}^{p} \frac{1}{\hbar_{\alpha}} y_{\bar{x}_{\alpha}}, \quad \Lambda^{+} y=\sum_{\alpha=1}^{p} \frac{1}{\hbar_{\alpha}} y_{x_{\alpha}}
$$

In this case the iterational ADI scheme is

$$
\bar{y}-\tau \Lambda-\bar{y}=y^{h}+\tau \Lambda^{+} y^{k}+\tau f, \quad x \in \omega_{h, q} ; \quad \bar{y}=\mu(x) \text { for } x \in \gamma_{h},
$$

$y^{k+1}-\tau \Lambda^{+} y^{h+1}=\bar{y}+\tau \Lambda-\bar{y}+\tau f, \quad x \in \omega_{h, q}, \quad y^{h+1}=\mu(x)$ for $x \in \gamma_{h}$.

This scheme was first proposed by V.K. Saul'ev for solving the equation of heat conduction (with $p=1,2$ ); he called it the alternating method [21]. It is economic, since $O\left(1 / h^{p}\right)$ arithnetical operations are required for transition from $y^{k}$ to $y^{k+1}$.

Introducing the operators $A_{1} y=-\Lambda^{-} y, A_{2} y=-\Lambda^{+} y$ into the space of mesh functions specified on $\omega_{h, q}$ and vanisning on the mesh boundary $\gamma_{h}$, we can rewrite $(87)$ as

$$
\left(E+\tau A_{1}\right) \bar{y}=\left(E-\tau A_{2}\right) y^{k}+\tau f, \quad\left(E+\tau A_{2}\right) y^{k+1}=\left(E-\tau A_{1}\right) \bar{y}+\tau \tilde{f}
$$

The right-hand side $\tilde{f}$ takes account of the non-homogeneous boundary conditions and only differs from $f$ at boundary base-points of the mesh.

The operators $A_{1}, A_{2}$ are non-seltadjoint and positive - definite in $\Omega$. We evaluate $\delta$ and $\Delta$ for them.

Using Green's difference formula as in paragraph 3, Section 3, we get $\left(A_{\alpha} y, y\right)=0.5(A y, y), \alpha=1,2$. After simple working similar to that of paragraph 1, Section 3 , we get

$$
\left\|A_{\alpha} y\right\|^{2} \leqslant \frac{2 p}{q^{2} h^{2}}\left(A_{\alpha} y, y\right), \text { i.e. } \Delta=\frac{2 p}{q^{2} h^{2}} .
$$


Since $(A y, y) \geqslant\left(4 p / D^{2}\right)\|y\|^{2}$, we have

$$
\left(A_{a} y, y\right) \geqslant \frac{2 p}{D^{2}}\|y\|^{2}, \quad \delta=\frac{2 p}{D^{2}},
$$

where $D$ is the diameter of the region $G$. Hence it follows that

$$
\eta=\frac{8}{\Delta} \frac{q^{2} h^{2}}{D^{2}}
$$

Te now apply Theorem 2

$$
\rho \leqslant \frac{1-\sqrt{\eta}}{1+\sqrt{\eta}}=\frac{1-q h / D}{1+q h / D}, \quad \tau=\tau^{*}=\frac{D q h}{2 p} .
$$

Thus the same asymptotic formula as in paragraphs 1,2 (for scheme (64)) holds for the number of iterations $\nu(\varepsilon)$ in the case of scheme (86) (87), namely,

$$
v(8)=O\left(\frac{1}{h} \ln \frac{1}{8}\right)
$$

which is independent of the number of dimensions ( $p$ does not appear in the expression for $p$, see (88)). The total amount of computation for scheme (87) is $O\left(\frac{1}{h^{p+1}} \ln \frac{1}{8}\right)$.

Given any $p$, we can define $A_{1}, A_{2}$ by analogy with (86) for the nonselfadjoint problem

$$
A_{1} y=\sum_{a=1}^{p}\left(\frac{v_{\alpha}}{\hbar_{\alpha}} y_{\bar{x}_{\alpha}}-b_{\alpha y_{x_{\alpha}}}\right), \quad A_{2} y=\sum_{\alpha=1}^{p}\left(-\frac{v_{\alpha}}{\hbar_{\alpha}} y_{x_{\alpha}}-b_{\alpha}^{+} y_{\hat{x}_{\alpha}}\right) .
$$

The bound (88) still holds in this case. If the mesh $\omega_{h}$ is non-uniform at regular base-points and $h_{\alpha}\left(x_{i}\right)=h(h=$ const. $>0)$ everywhere in $\omega_{h}$, the bound (88) holds if $q h$ is replaced by $h$.

Note. Then comparing different iterational methods, in addition to the number of iterations, we need to consider the error resulting from rounding-off errors when determining each iteration. For instance, the method of successive substitutions is used for realizing scheme (64). Hence, as follows from N.S. Bakhvalov's bounds, an error $O\left(\varepsilon_{0} / h^{2}\right)$ occurs when finding $y^{k+1}$, where $\varepsilon_{0}$ is the rounding-off error. In the case of schene (87), this error obviously does not exceed $O\left(\varepsilon_{0} / h\right)$. As we have 
seen, schemes (64) and (87) are similar as regards the number of iterations. The iterational ADI scheme can be used to solve equations with variable coefficients.

Acknow ledgement. The author takes this opportunity to thank E.G. D' yakonov for editorial comment.

Translated by D.E. Brown

REFERENCES

1. D'YAKONOV, E.G. On soms difference schemes for solving boundary value problems, Zh. vȳchisl. Mat. mat. Fiz. 2, 1, 57-59, 1962.

2. D' YAKONOV, E.G. Difference schemes with a decomposed onr rator for multidimensional non-stationary problems, $2 h . v \bar{y} c h i s l$. Mat. mat. $F i z, 2,4,549-568,1962$.

3. SAMARSKII, A.A. Schemes of Improved accuracy for the multidimensional equation of heat conduction, $Z h$. vīchisl. Mat. mat. Fiz. 3, 5, $812-840,1963$.

4. SAMARSKII, A.A. On difference schemes for multidimensional differential equations of mathematical physics, Aplik. mat. 10, 2, 146 164, 1965 .

5. PEACEMAN, D.F. and RACHFORD, H.H. The numerical solution of parabolic and elliptic equations, J. Soc. ind. appl. Math. No. 1, $28-$ 46. 1955 .

6. DOUGLAS, J. and RACHFORD, H. H. On the numerical solution of heat conduction problems in two and three space variables. Trans. Am. math. Soc. 82, 2, $421-439,1956$.

7. YANENKO, N.N. On a difference method for the multidimensional equation of heat conduction, Dokl. Akad. Nauk SSSR, 125, 6, 1207 1210,1959 .

8. Samarski, A.A. On the additivity principle for constructing economic difference schemes, Dokl. Akad. Nauk SSSR, 165, 6, 1253 - 1256, 1965 .

9. SAMARsKII, A.A. On the economic difference method for solving the 
multidimensional parabolic equation in any region, $Z h$. viychisl. Mat. mat. Fiz. 2, 5, $787-811,1962$.

10. SAMARSKII, A.A. On the theory of difference schemes, Dokl. Akad. Nauk SSSR, 165, 5, 1007 - 1011, 1965.

11. SAMARSKII, A.A. On an economic algorithm for numerical solution of a system of differential and algebraic equations, $Z h, v \bar{y} c h i s l$. Mat. mat. Fiz, 4, 3, 550-584, 1964.

12. MarciluK, G.I. and sultangazin, U.M. On the convergence of the decomposition method for the radiation transport equation, Dokl. Akad. Nauk SSSR, 161, 1,65-69, 1965.

13. MarchuK, G.I. and sultangazin, U.M. On proving the decomposition method for radiation transport equations, $Z h . v \bar{y} c h i s l$. Mat. mat. $F_{i z} .5,5,852-863,1965$.

14. PEARCY, C. On convergence of alternating direction procedures, Num. Wath. 4, 2, 172 - 176, 1962 .

15. DOUGLAS, J, and PEARCY, C.M. On convergence of alternating direction procedures in the presence of singular operators, Num. Math. 5 , 2, 175 - 184. 1963.

16. KANTOROVICH, L.V. and AKILOV, G.P. Functional Analys is in Normed Spaces (Funktsional'nyi analiz $v$ normirovannykh prostranstvakh). Fizmatgiz, Moscow, 1959.

17. WACHSPRESS, E.L. and HABETLER, G.T. An alternating direction implicit iteration technique, J. Soc, ind. appl. Math., 8, 403-424, 1960.

18. WACHSPRESS, פ.L. Optimum alternating direction implicit iteration parameters for a model problem, J. Soc. ind. appl. Math. 10, 2, 339 - 350, 1962.

19. SAMARSKII, A.A. Locally uniform schemes with non-uniform meshes, Zh, vychisl. Mat. mat. Fiz. 3, 3, 431-466, 1963.

20. SAMARSKII, A.A. On monotonic difference schemes for elliptic and parabolic equations in the case of a non-selfadjoint elliptic operator, $Z h$. vȳchisl. Mat. mat. Fiz. 5, 3, $548-551,1965$.

21. SAUL'EV, V.K. Integration of Parabolic Equations by the Mesh Method (Integrirovanie uravnenii parabolicheskogo tipa metodom setok). Fizmatgiz, Moscow, 1960. 